\title{
Detection of Hepatomegaly by Automated-Diagnosis Method based on Artificial Neural Network
}

\author{
Bratati Roy \\ Research officer \\ Hajee Mohammad \\ Danesh Science and \\ Technology University
}

\author{
Taskin Noor Turna \\ Lecturer, ICE \\ Pabna University of \\ Science and Technology
}

\author{
Md. Ebrahim Hossain \\ Lecturer, CSE \\ Leading University, Sylhet
}

\author{
Mst. Alema Khatun \\ Lecturer (CSE), BBA \\ Dhaka Commerce \\ College
}

\begin{abstract}
Diagnosis sonography (ultrasonography) is an ultrasoundbased diagnostic imaging technique which is used for visualizing internal body structures including tendons, muscles, joints, vessels and internal organs for possible pathology. In Bangladesh, there is no formal training for the sonographer, that's why sometimes there is a great possibility to make mistakes to find out the exact disease. Sonography is done manually. Sometimes the disease suggested by doctor and the diagnosis report that is done by the sonographer are not the same, for this reason the patient may be confused about his or her actual disease. To solve all these problems an automatic diagnosis tool is needed to get the required result of the required patient. Here, proposed an artificial neural network based model for automated diagnosis of hepatomegaly from ultrasound images to get an automated result which is appropriate for anyone.
\end{abstract}

\section{Keywords}

Diagnosis, ultrasound, Hepatomegaly, artificial neural network,

\section{INTRODUCTION}

In this work, artificial neural network based self-diagnosis method in ultrasound imaging is proposed. Ultrasonography is a medical imaging technique that uses high frequency sound waves to view soft tissues such as muscles and internal organs. Because ultrasound images are captured in real-time, they can show movement of the body's internal organs as well as blood flowing through blood vessels. Pattern recognition plays a great role to find an actual result than image processing. Pattern recognition is a process that takes in raw data and makes an action based on the feature of the pattern. The patterns of ultrasonic echoes from objects contain information about the geometric shape, size, orientation and the surface material properties of the reflector. Here a method of pattern recognition has been proposed to find automated results from ultrasound images by using artificial neural networks.

An ultrasound-based diagnostic imaging technique is diagnostic sonography (ultrasonography) which is used for visualizing internal body structures including tendons, muscles, joints, vessels and internal organs for possible pathology or lesions. Ultrasonography of pregnant women is called obstetric sonography, and now it is widely used. In the language of physics, 'ultrasound' refers to sound waves with a frequency too high for humans to hear. Ultrasound images (sonograms) are produced from sending a pulse of ultrasound into tissue with the help of an ultrasound transducer (shortly called probe). The sound reflects and echoes off parts of the tissue; this echo is recorded and displayed as an image to the operator. Different types of images can be formed using ultrasound. The most well-known type is a B-mode image, which displays a two- dimensional cross-section image. Besides, other types of images can display blood flow, motion of tissue over time, the location of blood, the presence of specific molecules, the stiffness of tissue, or the anatomy of a three-dimensional region. Ultrasound can also be used therapeutically, which helps to break up gallstones and kidney stones or to heat and destroy diseased or cancerous tissue [1].

\section{RELATED WORKS}

Few research works are for recognizing ultra-sonogram patterns ([2], [3], [4], [5], [6], [7]). In [2], a novel fuzzy feature extraction method to encode local texture which extends the local binary pattern approach by incorporating fuzzy logic in the representation of local patterns of texture in ultrasound images is proposed. The authors have evaluated Fuzzy Local Binary patterns For Ultrasound Texture Characterization. Optimize high dimensional input space and excellent generalization performance has been considered. In [3], a method to access the value of pattern recognition for the ultrasound diagnosis of borderline ovarian tumors (BOT) is proposed. The authors have evaluated Accuracy of Ultrasound Subjective 'Pattern recognition' for the Diagnosis of borderline ovarian tumors. In the paper [4], a method considering an automated detection and measurement method for nuchal organization (NT) is proposed. The authors have evaluated ultrasonic marker pattern recognition and measurement using artificial neural networks. Optimize local measurement of intensity, edge strength and continuity extracted and became the weighted terms for thickness calculation. Paper [5] presents a method considering a good communication system in order to give the eletro-oculogram organized at as a result of eye movement and found the optimum positions for the electrodes for the communication system. The authors have evaluated pattern recognition of eye movement. Optimize the signal of two eye movements taken with a MINDSET MS-100 system. Paper [6] presents a method of pattern recognition of ultrasound brain-stem images based on the PCA (principal component Analysis) method that is a very useful and known method for image processing. The authors have evaluated the image recognition of brain-stem ultrasound images by using a neural network based on PCA. Optimize interdisciplinary characters between medicine and computing and also optimize ultrasound modality that is best for brain scanning. Paper [7] proposed a method based on artificial neural networks that provides a decision support system to identify three main heart diseases: mitral stenosis, aortic stenosis and ventricular septal defect and the system deals with an encouraging opportunity to develop an operational screening and testing device for heart disease diagnosis and can deliver great assistance for clinicians to make advanced heart diagnosis. The authors have evaluated Innovative Artificial Neural Networks-Based Decision Support System for Heart Diseases Diagnosis. Optimize medical 
physicians to diagnose heart sound signals and check the upnormality and also the method provide innovative diagnostic tools to classify the heart disease.

\section{METHODOLOGY}

In an ultrasound examination, a transducer is placed directly on the skin or inside a body opening. A thin layer of gel is applied to the skin so that the ultrasound waves are transmitted from the transducer through the gel into the body. The ultrasound image is produced due to the reflection of the waves off of the body structures. The strength (amplitude) of the sound signal and the time it takes for the wave to travel through the body provide the information necessary to produce an image. Piezo electric transducer is produced by a sound wave by an encased in a plastic housing. The transducer gets its desired frequency from the ultrasound machine and the frequency is produced by strong and short electric pulses from the ultrasound machine. The frequency range is between 1 and $18 \mathrm{MHz}$. Older technology transducers mainly focused on beams with physical lenses. Phased array techniques are used by newer technology transducers which enable the ultrasound machine to change the direction and depth of focus. The sound is focused by the shape or complex set of control pulses or a lens in front of the transducer, from the ultrasound scanner. This focusing produces a wave which is arc-shaped at the face of the transducer. The wave is travelled inside the whole body and at a desired depth it comes into a focus. Materials that are on the face of the transducer enable the sound to be transmitted efficiently inside the whole body. Beside a water-based gel is placed in between the patient's skin and the probe. Between different tissues the sound wave is partially reflected from the layers or scattered from smaller structures. There are acoustic impedance changes in the body as sound is reflected anywhere: e.g. blood cells in blood plasma, small structures in organs, etc. Sometimes some of the reflections return to the transducer. The sound wave which is returned to the transducer results in the same process to send the sound wave except in reverse. The transducer vibrates by the returned sound wave and it turns the vibrations into electrical pulses that travel to the ultrasonic scanner to be processed and transformed into a digital image. [8]

As the ultrasonic scanner determines these two things, it can determine which pixels in the image to light up at its required intensity.

The digital image that is produced may be explained by using a blank spreadsheet as an analogy. In the first picture a long, flat transducer at the top of the sheet. Into the 'columns' of the spreadsheet (A, B, C, etc.) send some pulses. Carefully listen at each column which may return any echoes. As an echo is heard, it can be noted how long it took for the echo to return. The longer the wait, the deeper the row $(1,2,3$, etc.). Brightness setting for that cell (white for a strong echo, black for a weak echo, and varying shades of grey for everything in between.) is determined by the strength of the echo. All the echoes are recorded on the sheet, to form a gray scale image. Fig. 1is the flow chart of the proposed model.

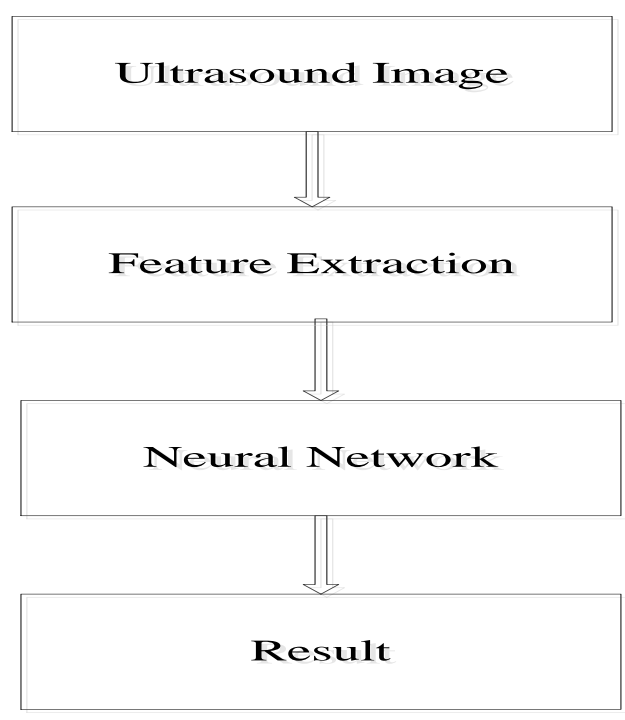

Fig 1: proposed model

High-frequency sound waves are used in ultrasound imaging to view soft tissues as muscles and internal organs of the body. As real-time is used when ultrasound images are captured, the movement of the body's internal organs as well as blood flow through blood vessels can be easily seen. The problems in the liver, heart, kidney or the abdomen can be detected by ultrasound scans. Higher frequencies provide better quality images, but this higher frequency is absorbed by the skin and other tissue, as a result they cannot penetrate like lower frequencies. The image quality is inferior though the lower frequencies can penetrate deeper. When an ultrasound scan is performed a sonogram of the image is produced. Though ultrasound travels through soft tissue and fluids, it bounces back off denser surfaces. Ultrasound also travels through blood, such as in the heart chamber, but much of it forms an echo when hitting a heart valve. Ultrasound will travel straight through when there are no solid gallstones in the gallbladder, but if there are any stones in the gallbladder, ultrasound will bounce back from them. The more bouncing back depends on the density of the object that ultrasound hits. Ultrasound images get its features varying shades of gray reflect different densities by the echo.

Abdominal Sonography (gastroenterology) - The images of the spleen, kidneys, bile ducts, gall bladder, liver, aorta, inferior vena cava, pancreas and other solid organs located in the abdomen are seen by abdominal sonography.

\subsection{Feature Extraction}

Feature extraction in image processing is an initial set of measured data and builds derived values which are intended to be informative, non-redundant, facilitating the subsequent learning and generalization steps, sometimes in some cases it leads to better human interpretations. Feature extraction and dimensionality reduction is related. When an algorithm contains input data which is too large to be processed and it is considered to be redundant then it can be transformed into a reduced set of features (also named features vector). This whole process is called feature extraction. The features which are extracted contain the relevant information from the input data, so that the task can be easily performed by using this reduced representation instead of the complete initial data. Feature extraction reduced the amount of resources required to describe a large set of data. Feature extraction is a method of constructing combinations of the variables to get rid of the problems and also to describe the data with sufficient accuracy. 
For detecting abnormalities of an object in case of geometrical shape, size and color feature extraction method is also used.

\subsection{Neural Network}

Artificial neural networks (ANNs) are a set of statistical learning algorithms inspired by biological neural networks both in machine learning and cognitive science and are used for estimation of functions that can depend on a large number of inputs and are in general unknown. Some of relevant characteristics of artificial neural network is it can able to learn how to do tasks based on the data given for training or initial experience, it can also can create its own organization or representation of the information it receives during learning time, it performs its computation in parallel way and special hardware devices are being designed and manufactured which take advantage of this capability. By artificial neural networks some network capabilities may be retained even with major network damage. [9]

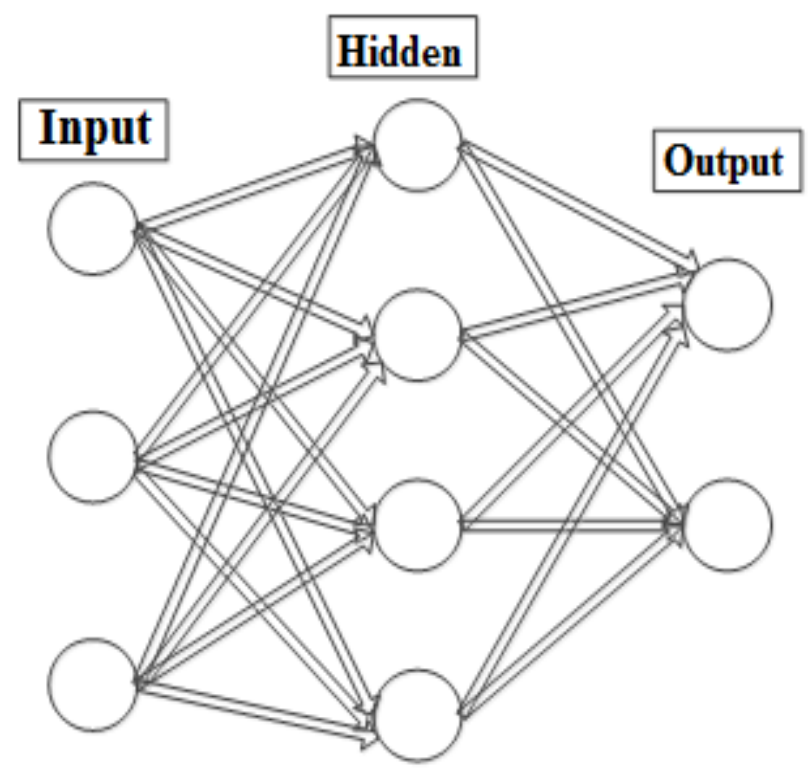

Fig 2: Artificial Neural Network [9]

An artificial neural network is interconnected by a huge collection of nodes, akin to the vast network of neurons in a brain. Here, each circular node is the representation of an artificial neuron and an arrow represents a connection from the output of one neuron to the input of another neuron. Neural networks and biological neural networks are similar in performing functions collectively and in parallel by the units, rather than there being a clear delineation of subtasks to which various units are assigned. In modern software implementations of artificial neural networks are largely abandoned for a more practical approach based on statistics and signal processing. In some of these systems, neural networks or parts of neural networks (like artificial neurons) form components in larger systems that combine both adaptive and non-adaptive elements.

\section{SYSTEM MODEL}

\subsection{Perimeter}

In the case of normal liver the edge is thin and firm and emerges to the lower edge of the right rib cage. On the other hand, an enlarged liver is caused by liver disorders related to excessive alcohol use, congestive heart failure, glycogen storage disease, viral hepatitis, liver cancer, and steatosis (fat in the liver). Large variety of other disorders, such as mononucleosis or tumor metastases from other primary cancers in the body may also be the reason for enlarged liver.

\subsection{Area}

The size of a normal liver for women is $7 \mathrm{~cm}$ and that for men is $10.5 \mathrm{~cm}$. When diagnosis of a liver if the size exceeds 2 to 3 $\mathrm{cm}$ larger, then the liver is marked to be abnormal and then the sonographer advices the patient to consult with the doctor.

\subsection{No. of objects}

After canny edge detection of a normal liver and enlarged liver here the number of white objects of enlarged liver is high compared to the no of objects of the normal liver. Here, the no of white objects are the shape of enlarged and normal liver.

\subsection{Noise reduction by smoothing}

Image that contained noise is smoothed by convolving the input image I $(i, j)$ with Gaussian filter G. Mathematically, the smooth resultant image is given by

$$
F(i, j)=G^{*} I(i, j)
$$

This is simpler as compared to the sobel operator but it is noise sensitive.

\subsection{Finding gradients}

Here the detection of the edges where the change in gray scale intensity is obtained maximum. Required areas are determined with the help of gradient of images. Sobel operator is used to determine the gradient at each pixel of a smoothened image. Sobel operators in $\mathrm{i}$ and $\mathrm{j}$ directions are given as

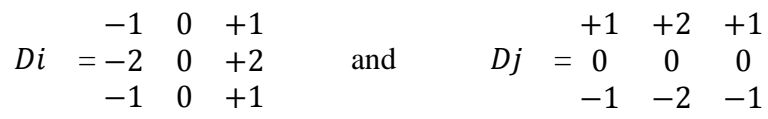

These sobel masks are convolved with a smoothed image and giving gradients in $i$ and $j$ directions.

$$
G i=D i * F(i, j), \text { And } c=D j * F(i, j)
$$

Therefore edge strength or magnitude of gradient of a pixel is given by

$$
\mathrm{G}=\sqrt{ } G i^{2}+G j^{2}
$$

The direction of gradient is given by $\theta=\arctan \left(\frac{G i}{G j}\right)$

Giand $G j$ are the gradients in the i- and j-directions respectively.

\subsection{Non maximum suppressions}

Non maximum suppression is used to preserve all local maxima in the gradient image, and deleting everything else these results in thin edges. For a pixel M (i, j): Firstly round the gradient direction $\theta$ nearest $45^{\circ}$, then compare the gradient magnitude of the pixels in positive and negative gradient directions i.e. If the gradient direction is east then compare with the gradient of the pixels in east and west directions say E $(i, j)$ and $W(i, j)$ respectively. If the edge strength of pixel $M$ $(i, j)$ is largest than that of $E(i, j)$ and $W(i, j)$, then preserve the value of gradient and mark $M(i, j)$ as edge pixel, if not then suppress or remove.

\subsection{Hysteresis Thresholding}

The output of non-maxima suppression still contains the local maxima created by noise. Instead of choosing a single threshold (for avoiding the problem of streaking) two thresholds $t_{\text {low }}$ and $t_{\text {high }}$ are used.For a pixel $\mathrm{M}(\mathrm{i}, \mathrm{j})$ having gradient magnitude $\mathrm{G}$ following conditions exists to detect pixel as edge: 
- If $\mathrm{G}<t_{\text {low }}$ than discard the edge.

- If $\mathrm{G}>t_{\text {high }}$ than keep the edge.

- If $t_{\text {low }}<\mathrm{G}<t_{\text {high }}$ and any of its neighbors in a $3 \times 3$ region around it have gradient magnitudes greater than $t_{\text {high }}$, keep the edge.

- If none of pixel (x, y)'s neighbors have high gradient magnitudes but at least one falls between $t_{\text {low }}$ and $t_{\text {high }}$, search the $5 \times 5$ region to see if any of these pixels have a magnitude greater than thigh. If so, keep the edge.

- $\quad$ Else, discard the edge.

\section{ANALYSIS AND RESULT}

Firstly there's need to find out the edge by any standard edge detection algorithm (canny, X etc.). After that, find out the area of normal image and the area of fatty image. And also find out the number of objects determined by canny edge detection algorithm [10].

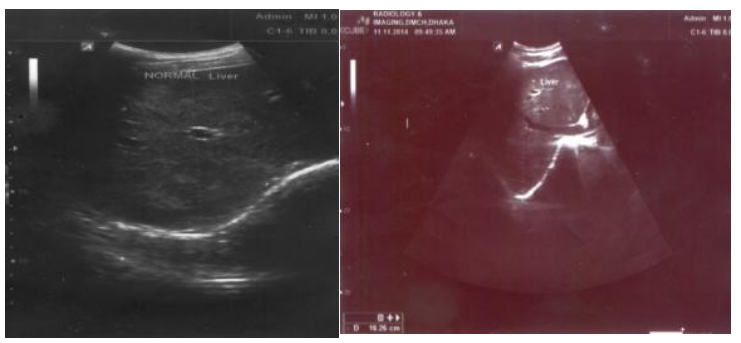

Fig 3: (a). Normal Liver with $11.98 \mathrm{~cm}$ (b). Fatty liver with $16 \mathrm{~cm}$

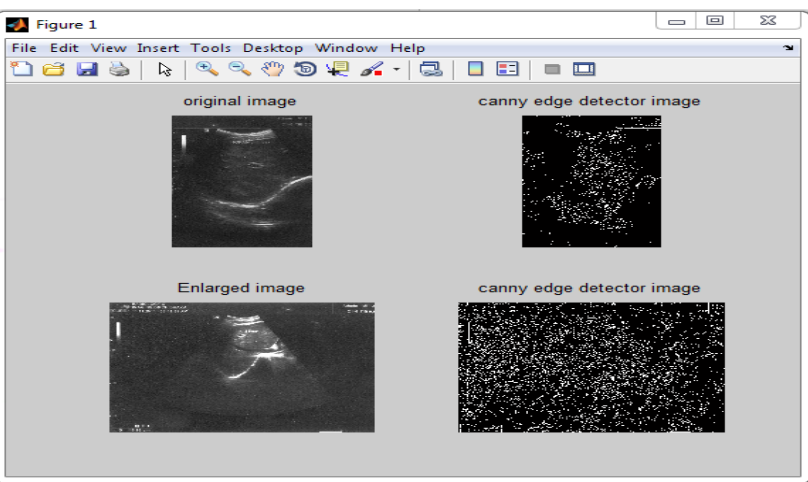

Fig 4: result using Canny edge detection algorithm

First the input value and target value is imported to the workspace to compare later this value in a neural network tool. For this some random data needed to classify the network and then import the data to the network. Fig. 5 and Fig. 6 shows the input and target value generated by matlab.

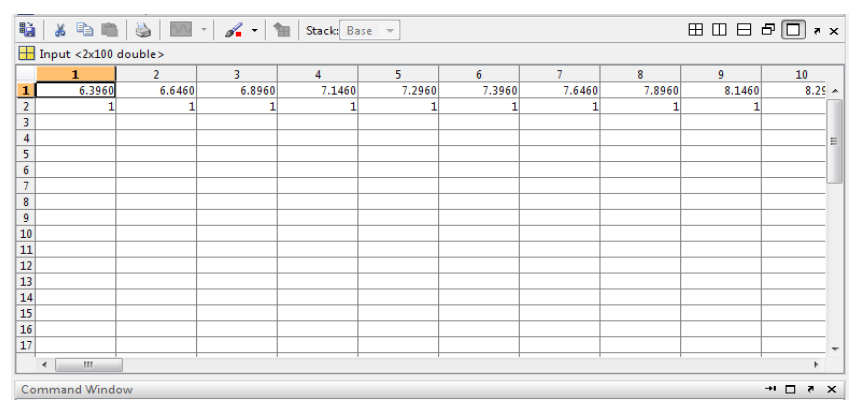

Fig 5: Input Value Generated By MATLAB

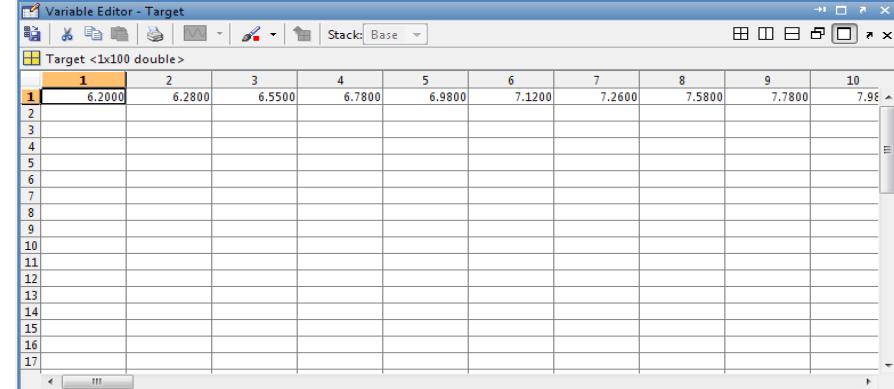

Fig 6: Target Value Generated By MATLAB

After importing input and target data a feed-forward backprop network is created with input data and target data, training function TRAINLM, adaptation learning function LEARNGDM, performance function MSE, transfer function TANSIG and number of layer of 2. Fig 7 and Fig. 8 show the neural network.

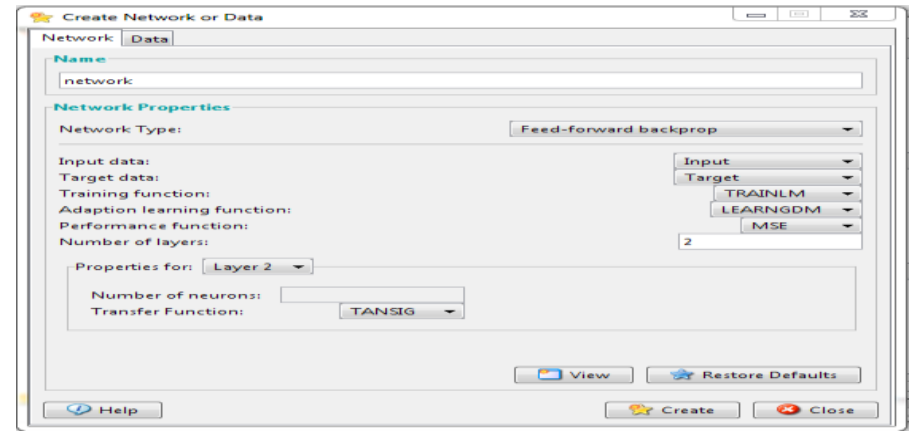

Fig 7: Creating neural network

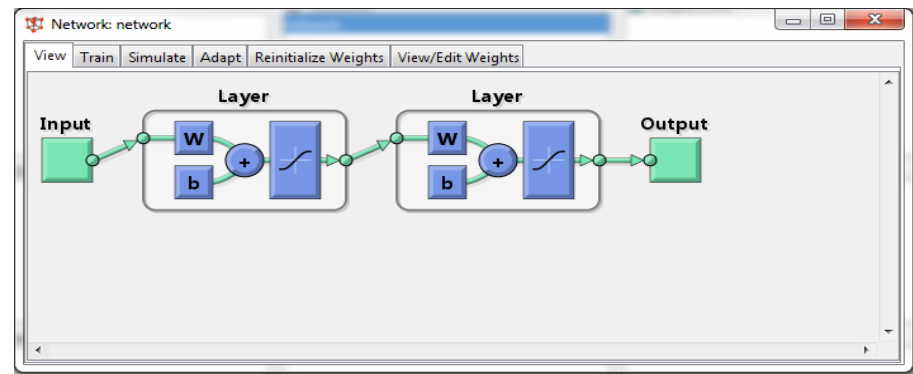

Fig 8: Neural network

Then the whole network is trained with a learning rate of $(\mathrm{mu})$ 0.001 .

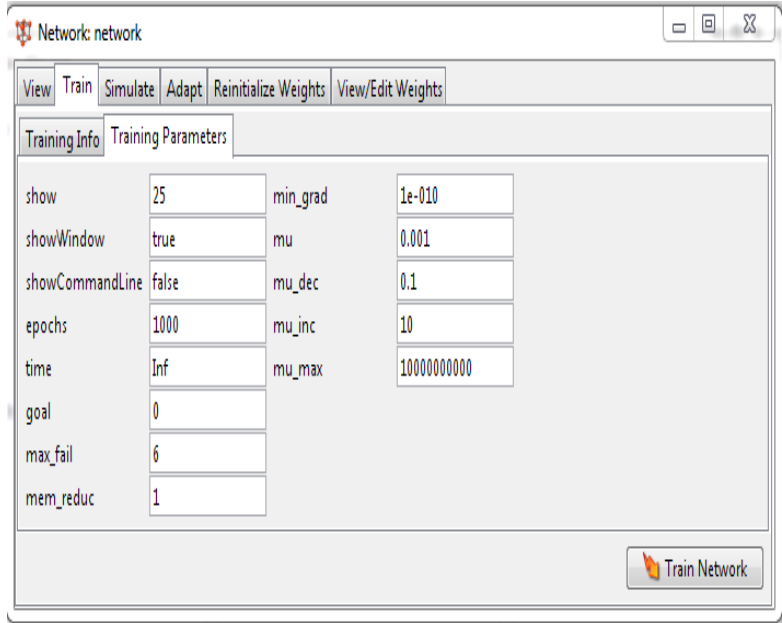

Fig 9: Training parameters 
After training the network is shown in Fig 10.

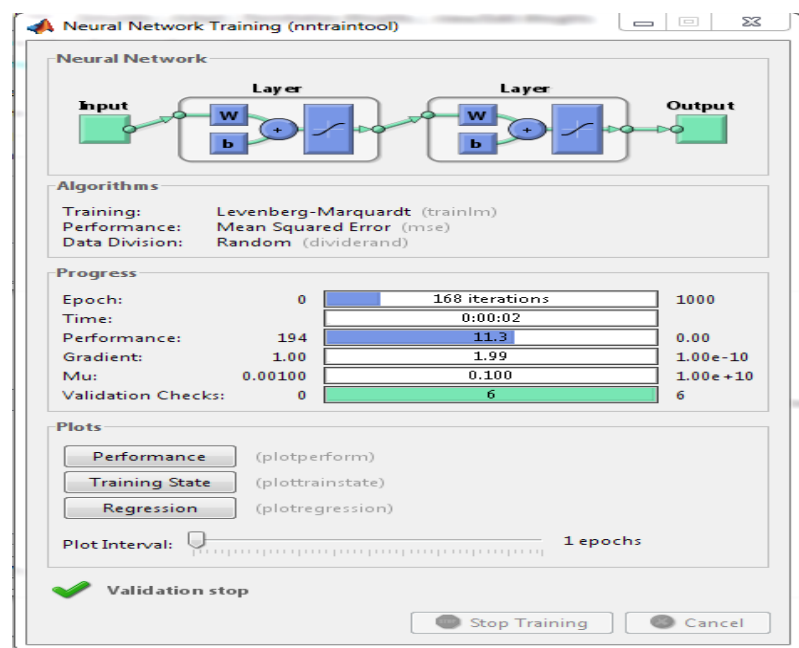

Fig 10: Network after Training

The performance graph of the network at a low learning rate is in Fig 11.

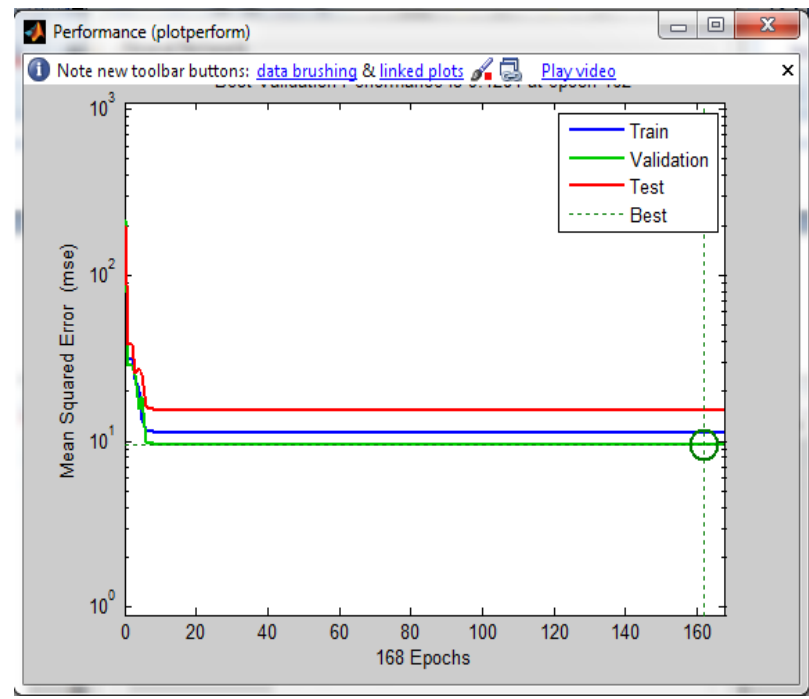

Fig 11: Performance graph in low learning rate

Table 1: Target data, data output and error percentage in case of learning rate 0.001

\begin{tabular}{|c|c|c|}
\hline $\begin{array}{c}\text { Targe } \\
\text { t data }\end{array}$ & $\begin{array}{c}\text { Data } \\
\text { Output } \\
\mathbf{s}\end{array}$ & $\begin{array}{c}\text { Error } \\
\text { Percentag } \\
\mathbf{e}\end{array}$ \\
\hline 6.2 & 6.3593 & $2.56 \%$ \\
\hline 6.28 & 6.4612 & $2.88 \%$ \\
\hline 6.55 & 6.786 & $3.06 \%$ \\
\hline 6.78 & 6.9135 & $1.96 \%$ \\
\hline 6.98 & 7.005 & $0.35 \%$ \\
\hline 7.12 & 7.7866 & $9.36 \%$ \\
\hline 7.26 & 7.9498 & $9.50 \%$ \\
\hline 7.58 & 8.058 & $6.30 \%$ \\
\hline 7.78 & 8.5846 & $10.34 \%$ \\
\hline 7.98 & 8.8375 & $10.74 \%$ \\
\hline
\end{tabular}

In the Fig 12 the target data for first input is 6.2 and desired output is 6.3593 . So here the method only has $2.56 \%$ error which is negligible. In this way it can give the approximate desired result of diagnosis with learning rate 0.001 .

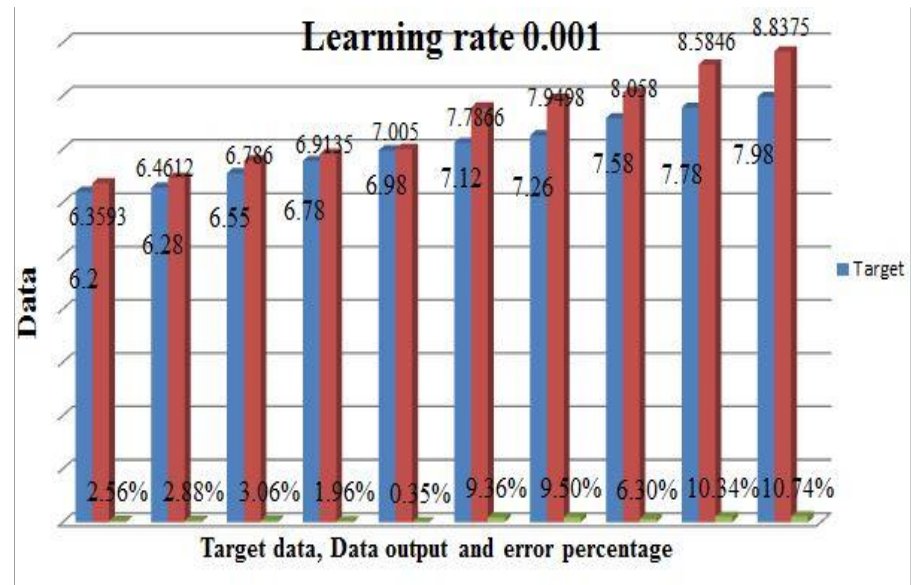

Fig 12: Graph with learning rate 0.001

2 Performance (plotperform) 近回|x

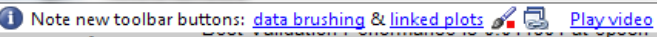

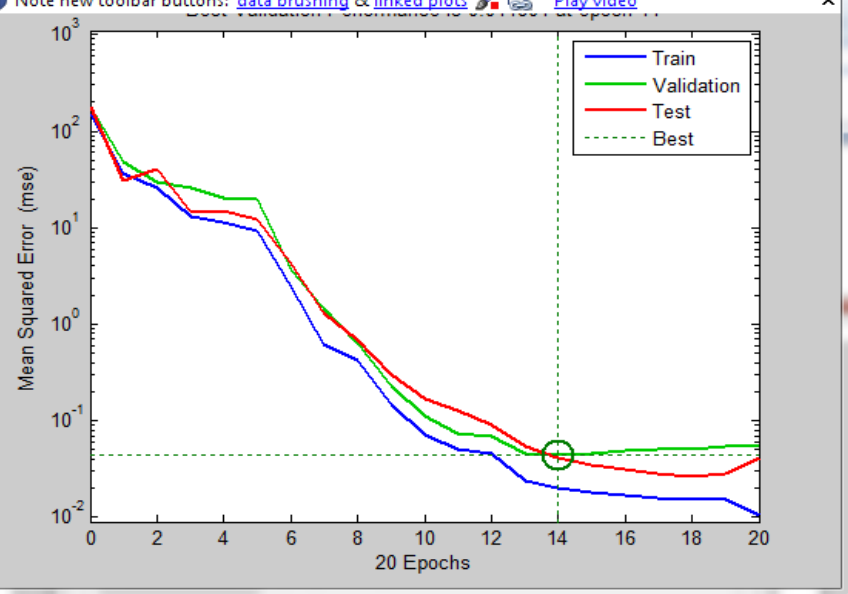

Fig 13: Performance Graph with learning rate 0.001

Table2: Target data, data output and error percentage in case of learning rate 0.01

\begin{tabular}{|c|c|c|}
\hline $\begin{array}{c}\text { Target } \\
\text { data }\end{array}$ & $\begin{array}{c}\text { Data } \\
\text { Outputs }\end{array}$ & $\begin{array}{c}\text { Error } \\
\text { Percentage }\end{array}$ \\
\hline 6.2 & 6.9691 & $12.40 \%$ \\
\hline 6.28 & 7.642 & $20.79 \%$ \\
\hline 6.55 & 7.827 & $19.49 \%$ \\
\hline 6.78 & 7.9463 & $17.20 \%$ \\
\hline 6.98 & 8.0292 & $15.03 \%$ \\
\hline 7.12 & 8.2478 & $15.83 \%$ \\
\hline 7.26 & 8.7285 & $20.22 \%$ \\
\hline 7.58 & 8.8823 & $17.18 \%$ \\
\hline 7.78 & 8.9869 & $15.51 \%$ \\
\hline 7.98 & 9.2541 & $15.96 \%$ \\
\hline
\end{tabular}


Learning rate 0.01

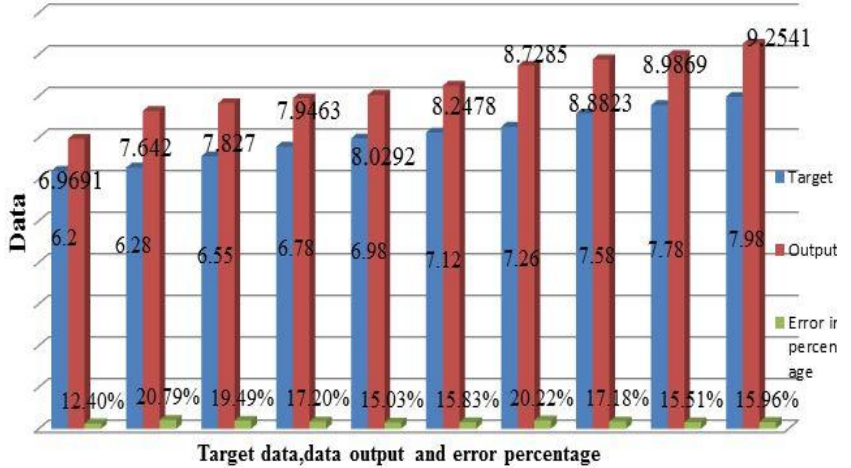

Fig 14: Graph with learning rate 0.01

In Table 2 and Fig 14 the target data for first input is 6.2 and the desired output is 6.9691. So here the method has $12.40 \%$ error which is higher compared to the proposed method. That's why it cannot give the approximate desired result of diagnosis with learning rate 0.01 . Fig 15 is the performance graph with learning rate 0.01 .

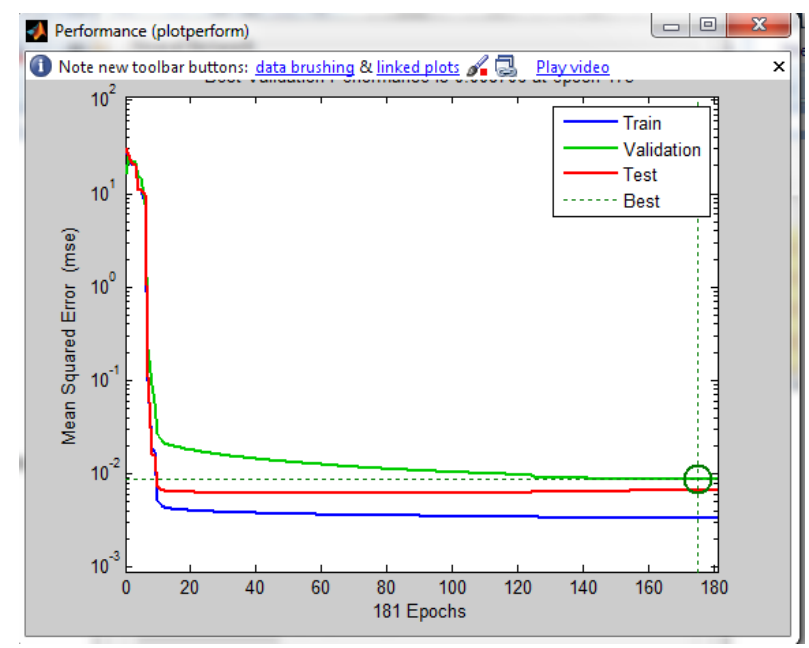

Fig15: Performance Graph with learning rate 0.01

In table 3 and Fig 16 the target data for first input is 6.2 and the desired output is 6.8424 . So here the method has an error of $10.36 \%$ which is also higher than the proposed method. In this way it also cannot give the approximate desired result of diagnosis with learning rate 0.1 .

Table 3: Target data, data output and error percentage in case of learning rate 0.1 .

\begin{tabular}{|c|c|c|}
\hline $\begin{array}{c}\text { Target } \\
\text { data }\end{array}$ & $\begin{array}{c}\text { Data } \\
\text { Outputs }\end{array}$ & $\begin{array}{c}\text { Error } \\
\text { Percentage }\end{array}$ \\
\hline 6.2 & 6.8424 & $10.36 \%$ \\
\hline 6.28 & 7.0617 & $12.44 \%$ \\
\hline 6.55 & 7.3016 & $11.47 \%$ \\
\hline 6.78 & 7.554 & $11.41 \%$ \\
\hline 6.98 & 7.9679 & $14.15 \%$ \\
\hline 7.12 & 8.0716 & $13.36 \%$ \\
\hline 7.26 & 8.8364 & $21.71 \%$ \\
\hline
\end{tabular}

\begin{tabular}{|c|c|c|}
\hline 7.58 & 9.0858 & $19.86 \%$ \\
\hline 7.78 & 9.5808 & $23.14 \%$ \\
\hline 7.98 & 9.9752 & $25 \%$ \\
\hline
\end{tabular}

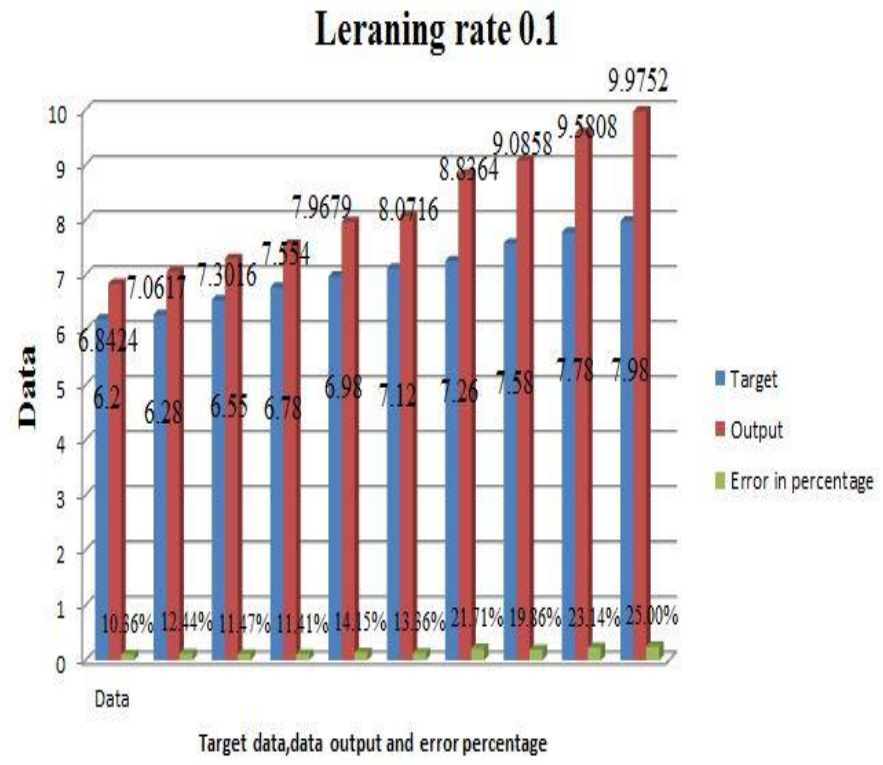

Fig 16: Graph with learning rate 0.1

\section{CONCLUSION}

The performance of ultrasonography is carried out by individuals without any formal training. That's why sometimes the diagnostic result is not perfectly recognized. To get the correct result the trainer should perform some training courses perfectly which is costly and not available everywhere. Here in this paper a self-diagnosis method is proposed in ultrasound imaging considering three parameters as perimeter, area and number of objects of ultrasound image. In this paper, the ultrasound image of normal liver and enlarged liver has been considered. After that considering an artificial neural network the data with different learning rates has been trained and tested. As well as the lower the learning rates the higher the performance rate of the method.

\section{REFERENCES}

[1] Ultrasonography. Retrieved date 18/10/18 Retrieved from https://www.fda.gov/radiation-emitting-products/medicalimaging/ultrasound-imaging.

[2] Dimtris K. Lakovidis, Eystratios G. Keramids, DimtrisMaroulis, "Fuzzy Local Binary patterns For Ultrasound Texture Characterization ", $3^{\text {rd }}$ European Support Framework-PENED 2003 (grant no. 03-ED662),2003.

[3] J.Yazbek, K. S. Raju, J. Ben-NaGI, T. Holland, K. Hillby, D. Jurkovic,"Accuracy of Ultrasound Subjective 'Pattern recognition' For The Diagnosis Of Borderline Ovarian Tumors", published online in Wiley Interscience,DOI:10.1002/uog.4002,2007.

[4] Eko Supriyanto, Laikhin Wee, Too Yuen Min, "Ultrasonic Marker Pattern Recognition And Measurement Using Artificial Neural Network", $9^{\text {th }}$ WSEAS International Conference On Signal Processing, ISSN:1790-5117, ISBN:978-954-92600-4-5,2005. 
[5] Jose De Jesus Rubio, Carlos Aviles, Raymundo Coello, Jose Francisco Cruz, Hector Rivero, "Pattern Recognition of Eye Movement", IEEE. 2009.

[6] Jiri Blahuta, Tomas Soukup, Petr Cermak,''The Image Recognition Of Brain-stem Ultrasound Images With Using a Neural Network Based on PCA', Issue 2,Volume 5,International Journal Of Applied Mathematics and Informatics, 2011.

[7] Sameh Ghwanmeh, Adel Mohammad, Ali Al-Ibrahim,"' Innovative Artificial Neural Networks-Based Decision Support System for Heart Diseases Diagnosis", Journal of
Intelligent Learning Systems and Applications, August 2013.

[8] Ultrasound. Retrieved date 18/10/18. Retrieved from https://www.medicalnewstoday.com/articles/245491\#uses

[9] Artificial Neural Network. Retrieved date 18/10/18 Retrievedfromhttps://en.wikipedia.org/wiki/Artificial_neu ral_network

[10] Canny Edge Detection. Retrieve date 20/10/18. Retrieved fromhttp://fourier.eng.hmc.edu/e161/lectures/canny/node1 .html. 